\title{
Application of Factor Analysis to Skin Temperature Distribution
}

\author{
by \\ H. Maruyama and M. Momiy ama \\ Meteorological Rescarch Institute \\ (Received August 13, 1951)
}

\begin{abstract}
We have applied the method of factor analysis to the problem of reducing the number of the otserving parts required for the calculation of the average skin temperature of the human body.

We have analyzed the observed data on the twenty-two parts adding to them the air temperatue and the age which are considered to influence the skin temperature. It has been thus, found that the observed values were rather well collected at every part, and that air temperature is actually one of the factors which controlled skin temperature.
\end{abstract}

\section{Introduction}

In the Research Committee on Climate physiology, they use the temperatue values of twenty-two parts of skin for the calculation of the average skin temperature of human body. For practical application, however, it is difficult to measure the temperature at so many points, nor so high an accuracy is needed either, so that the problem of reducing the number has been suggested. Among the physiologists in the United States, too, it is said recently eight parts are enough. [1]

To solve this problem objectively, it is most important to know what kinds of common factors control skin temperature and to what extent. For this purpose, the method of factor analysis seems to be very excellent. This analysis, however, has a defect of having no theor $y$ of exact sampling at present.

\section{Method of factor analysis}

The method of factor analysis is shown briefly in the following. Supposing that the observed value $X_{j}(j=1,2, \cdots \cdots, n)$ by the judgement $j(j=1,2, \cdots \cdots, n)$ - in this case the judgements equal to the skin parts---is estimated as normal distribution $N\left(m j, \sigma_{. j}^{2}\right)$,

$$
Z_{i j}=\frac{X_{j}-m_{j}}{\sigma_{j}}
$$

will submit to $N(0,1)$.

We put,

(1)

$$
Z_{j}=a_{j 1} F_{1}+a_{j 2} F_{2}+\cdots \cdots+a_{j_{m}} F_{m}+a_{j} U_{j}
$$


where the common factor is $F_{s}(s=1,2, \cdots \cdots, m)$, and the unique factor is $U_{j}$. And supposing that $\dot{F}_{s}$ and $U_{j}$ submit to $N(0,1)$, and that they are independent from each other, the following relationships hold.

$$
\begin{gathered}
\rho_{j k}=E\left(Z_{j} Z_{k}\right)=\sum_{s}^{m} a_{j s} a_{k s s} \\
\rho_{j j}=h_{j}{ }^{2}=\sum_{s}^{m}\left(a_{. j s}\right)^{2} \\
\rho_{Z}{ }_{j} k_{s}=a_{j s}
\end{gathered}
$$

When the sample size is large, we can approximate $m_{j}$ and $\sigma_{j}^{2}$ to be $\bar{x}_{j}$ (Sample mean) and $S_{j}{ }^{2}$ (Sample variance) which are calculated from the sample respectively and also we can approximate $\rho_{j k}$ to be sample correlation coefficient $r_{j k}$. Then from the correlation matrix, we can calculate the coefficient of comnon factor $F_{j s}$. Although there are various methods of factor analysis, the centroid solution is used in this study.

$Z_{j}$ is expressed by a point in $m$ dimension,

$$
p_{j}=\left(\alpha_{j 1}, \alpha_{j 2}, \cdots \cdots, a_{j_{m}}\right) \quad(j=1,2, \cdots \cdots, n)
$$

Determining the axis of the first factor as it passes through the centre of gravity of the point $n$, the coordinate of centre is expressed as follows;

$$
\left(\frac{1}{n} \sum_{j=1}^{n} a_{j 1}, O, O, \cdots \cdots, O\right)
$$

And then the following formulae hold,

$$
\begin{gathered}
r_{j k}=a_{j 1} \alpha_{k 1,}, \\
a_{, j 1}=\frac{\sum_{k i} r_{j /}}{\sqrt{\sum_{j} \sum_{h} \mathrm{r}_{j k}}},
\end{gathered}
$$

and the coefficient of the first factor of part $j$ is obtained. For $r_{j, j}=\left(h_{j}{ }^{2}\right)$, the maximum value in $\left|r_{j k}\right|$ in the row $j$ is used. The coefficient of the second factor can be obtained in the same way by using $\dot{r}_{j k}$,

$$
\dot{r}_{j k}=r_{j / c}-a_{j 1} \alpha_{k 1}
$$

In the same manner, the following coefficients are obtained. [2]

\section{Data}

For an example of practical analysis, the data on fifty persons and at the air temperature of $15^{\circ} \sim 20^{\circ} \mathrm{c}$, were extracted from the whole data observed under the same regulation as that of the Research Committee on Climate Physiology mentioned above.

In this case, we used twenty-two parts, namely, head (No.1), face (Nos. 2 and 3), neck (Nos. 4 and 5), breast (Nos. 6 and 8), abdomen (Nos. 9 and 10), loin (Nos. 11 and 12), upper arm (Nos. 14 and 15), fore arm (Nos. 16 and 17), hand (Nos. 18 and 19), thigh (Nos, 21 and 22), e.eg (Nos. 23 and 24), and foot (No.25). The ages of those fifty persons range from 20 years to 60 years old, having the 
mode near 25 years old.

\section{Results obtained and consideration.}

When we try factor analysis, it is the most difficult problem to know what the factor is. If the factor is presumed, we can recognize whether the estimation is true or not by calculation, putting the estimated factor into the judgements. When the estimation is true, fofmula (4) will be satisfied and the communality of the factors will be close to the unit 1 .

We tried factor analysis adding air temper ature and age, both as the estimated factors, to the observed skin temperature at thosetwenty-two parts. Namely, we analyzed the problem assuming the sample size was twenty-four. The 1eason why we took up those two factors as common factors is as follows; as for the air temperature, it controls greatly the skin temperatures of the naked parts while it hat dly does those of the covered parts: as for the age, ganerally speaking, the older the age is, the more conspicuosly high is the skin temperature of the upper legs and the lower are those of the other parts.

At first, we calculated the first factor $F_{1}$ and the socond factor $F_{2}$, but, as the remaining cor relation coefficients were found to be insignificant, we gave up the calculation. The coefficients of $F_{1}$ and $F_{2}$ are shown in the figure.

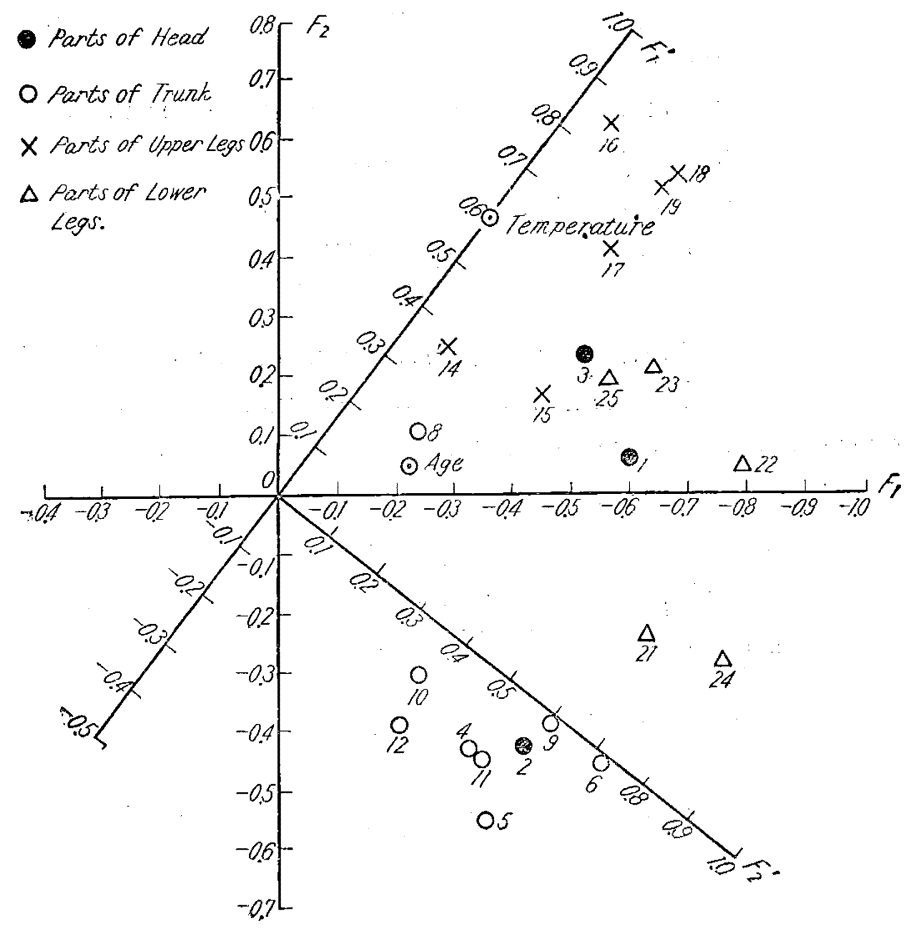

Factor Coefficients. (A Summary of the Research Committee on Climate Physiology ) 
It will be preferable to consider that age is not contained in the two factors estimated above, because the communality of age is 1ather small. As for the air temperature, though its communality seems not to be very near the unit 1 , it is considered to be one of the estimated factors. In the figure, the coefficients at each part are counted after rotating the coordinates, and then as the tendencies of variation of the counted coefficients considerably resemble those of the corretation coefficients between temperature and each part, the factor $F_{1}^{\prime}$ seems to be of temperature itself or of elements relating to temperature.

Though $F_{2}^{\prime}$ acts on the parts of trunk perceptively, it scarcely does on the parts of upper legs. While the first factor is one related to the radiation of body heat, - for instance, one which controls vasomoter, $-\mathrm{F}_{2}^{\prime}$ seems to be a factor related to the heat production of human body, that is, externally, it may be considered to be the condition of wearing.

The observed values on the conditions of each part are collected comparatively well, as is shown clearly in the figure. In any part of the trunk (neck, abdomen, and loin), the coefficient of $F_{2}{ }^{\prime}$ is small while that of $F_{2}{ }^{\prime}$ is relatively large excepting at No. 8. 'In the parts of upper legs (upper arms, before arms, and hands), the coefficient of $\mathrm{F}_{1}{ }^{\prime}$ is large while that of: $F_{2}{ }^{\prime}$ is small, and those of $F_{1}{ }^{\prime}$ and $\mathrm{F}_{2}{ }^{\prime}$ at the lower legs and at the head excepting at No. 2 show intermediate values. And, the conditions of the back (No.8) and the forehand (No. 2) are remarkable. The skin temperature of the back (the part right under the spina scapula along the scapula line) is not controlled so much by $F_{1}{ }^{\prime}$ and $F_{2}{ }^{\prime}$ as the other parts of the trunk. The reason of this fact, however, and also whether it is concerned on the whole back or only on this part are not obvious. For those two factors, the condition of the skin temperature of the forehead (the point $2 \mathrm{~cm}$ above the eyebrows, the middle of forehead) is the same as that of the trunk.

Acknowledgment-Lastly, we express our thanks to Dr, M. Masuyama who guided us kindly, and to the members of the Research Committee on Climate Physio ogy who offered us many precious data.

\section{References}

[1] Newburgh 1949: Physiology of heat regulation and Science of Clothing.

[2] Holzinger K.J. and Harman H.H. 1941: Factor Analysis, University of Chicago, Lllinois. 